\title{
Combined Semianalytical and Numerical Static Plate Analysis. Part 1: Formulation of the Problem and Approximation Models
}

\author{
Oleg Negrozov ${ }^{1,2}$, Pavel Akimov ${ }^{1,2, *}$, and Marina Mozgaleva ${ }^{1}$ \\ ${ }^{1}$ Moscow State University of Civil Engineering, Yaroslavskoe Shosse, 26, Moscow, 129337, Russia \\ ${ }^{2}$ Russian Academy of Architecture and Construction Sciences; 24-1, ul. Bolshaya Dmitrovka, \\ Moscow, 107031, Russia
}

\begin{abstract}
The distinctive paper is devoted to solution of multipoint (particularly, two-point) boundary problem of plate analysis (Kirchhoff model) based on combined application of finite element method (FEM) and discrete-continual finite element method (DCFEM). As is known the Kirchhoff-Love theory of plates is a two-dimensional mathematical model that is normally used to determine the stresses and deformations in thin plates subjected to forces and moments. The given domain, occupied by considering structure, is embordered by extended one. The field of application of DCFEM comprises fragments of structure (subdomains) with regular (constant or piecewise constant) physical and geometrical parameters in some dimension ("basic" dimension). DCFEM presupposes finite element mesh approximation for non-basic dimension of extended domain while in the basic dimension problem remains continual. FEM is used for approximation of all other subdomains (it is convenient to solve plate bending problems in terms of displacements). Discrete (within FEM) and discrete-continual (within DCFEM) approximation models for subdomains are under consideration.
\end{abstract}

\section{Introduction}

As is known the Kirchhoff-Love plate theory extends the Euler / Bernoulli beam assumptions to the two-dimensional case. Every significant measure of rotation, force, moment is evaluated with respect to the vertical deflection. The main kinematic assumption is that plane surfaces remain plane and perpendicular to the mid-surface of the plate $[7,10$, $15,17,18,21,26,34,38]$. Thus the Kirchhoff-Love theory predicts a zero distribution of shear stresses along the thickness. Therefor it can only be applied in problems where the variation of such stresses is expected to be small and their mean value does not deviate from 0 . Such can be considered the case of thin plates [12,23,25,27,28,32,37,42]. The distinctive paper is devoted to combined semianalytical and numerical static plate analysis. Solution of multipoint (particularly, two-point) boundary problem of plate analysis, based on combined application of finite element method (FEM) [11,13,14,19,22,24,36,39,40,41,43] and

* Corresponding author: pavel.akimov@gmail.com 
discrete-continual finite element method (DCFEM) [1,3-5,29,30,35,46] is under consideration. The given domain, occupied by considering structure, is embordered by extended one within so-called method of extended domain [2]. The field of application of DCFEM comprises fragments of structure (subdomains) with regular (constant or piecewise constant) physical and geometrical parameters in some dimension ("basic" dimension). DCFEM presupposes finite element mesh approximation for non-basic dimension of extended domain while in the basic dimension problem remains continual (corresponding correct analytical solution is constructed). FEM is used for approximation of all other subdomains $[6,8,9,16,31,33,44,45]$. Discrete (within FEM) and discrete-continual (within DCFEM) approximation models for subdomains are constructed. Besides, coupled multilevel approximation model for extended domain and brief information about software systems [20] and verification samples are presented in the second part of this paper.

\section{Formulation of the problem and approximation}

Let's consider multipoint (particularly, two-point) boundary problem of plate analysis within Kirchhoff model [12,23,25,27,28,32,37,42]. Several elements of corresponding notation system for corresponding two-point boundary problem are presented at figure 1.

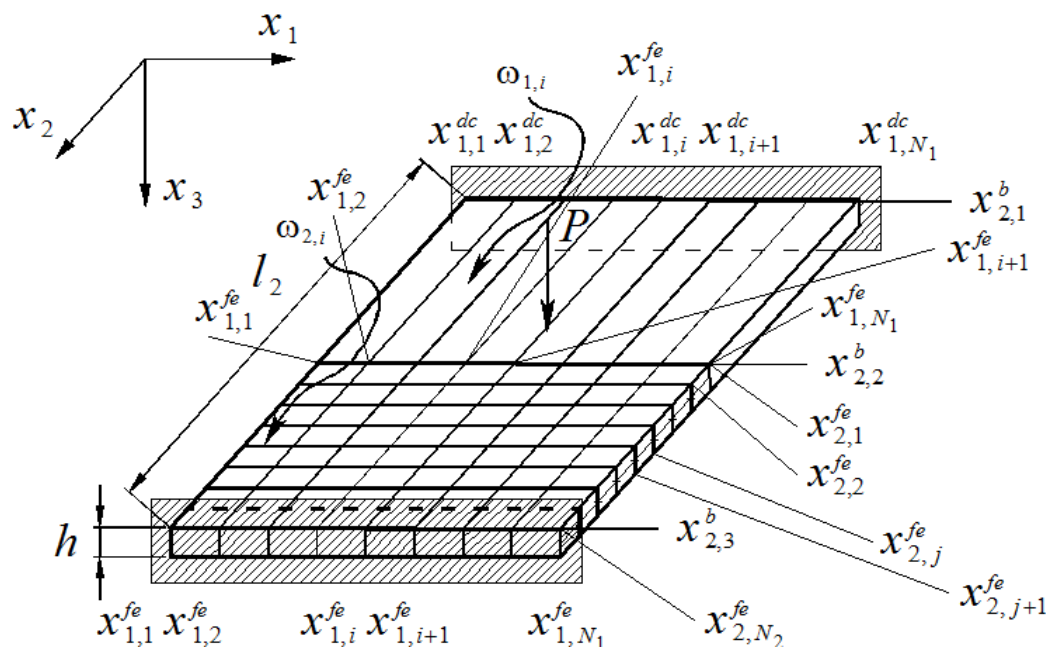

Fig. 1. Static analysis of thin plate.

Let's $\Omega$ be domain occupied by structure, $\Omega=\left\{\left(x_{1}, x_{2}\right): 0<x_{1}<l_{1}, 0<x_{2}<l_{2}\right\}$ or

$$
\Omega=\Omega_{1} \cup \Omega_{2}, \quad \Omega_{k}=\left\{\left(x_{1}, x_{2}\right): 0<x_{1}<l_{1}, x_{2, k}^{b}<x_{2}<x_{2, k+1}^{b}\right\}, \quad k=1,2,
$$

where $x_{1}, x_{2}$ are coordinates ( $x_{2}$ corresponds to basic dimension); $x_{2, k}^{b}, k=1,2, \ldots, n_{b}$ are coordinates of corresponding boundary points (cross-sections) along basic dimension (for mulipoint boudary peoblems $n_{b}>2\left(x_{2,1}^{b}=0, x_{2,2}^{b}=l_{2,1}, x_{2,3}^{b}=l_{2,1}+l_{2,2}=l_{2}\right) ; \Omega_{k}, k=1,2$ are subdomains of $\Omega$.

In accordance with the method of extended domain, proposed by Professor Alexander B. Zolotov [2], the given domain is embordered by extended one of arbitrary shape, particularly elementary. Let $\omega_{k}, k=1,2$ are extended subdomains, embordering subdomains $\Omega_{k} \subset \omega_{k}, k=1,2$, 


$$
\omega=\omega_{1} \cup \omega_{2}, \quad \Omega_{1} \subset \omega_{1}, \quad \Omega_{2} \subset \omega_{2} .
$$

Without loss of generality we suppose piecewise constancy of physical and geometrical parameters of one group of subdomains from (2) along coordinate $x_{2}$ ("basic" dimension). It is necessary to note that physical and geometrical parameters of structure can be changed arbitrarily along $x_{1}$. Thus, it is recommended to use DCFEM for approximation of these subdomains (discrete-continual design model is introduced).

Let physical and geometrical parameters within other group of subdomains from (2) are arbitrary varying. FEM $[44,45]$ can be used for approximation here. Combined application of DCFEM and FEM is advisable.

Operational formulation of the problem of plate analysis within Kirchhoff model and method of extended domain is used.

We can introduce the following notations for considering two-point boundary problem (Fig. 1): $x_{1, i}^{d c}, i=1,2, \ldots, N_{1}^{d c}$ are coordinates (along $x_{1}$ ) of nodes (nodal lines) of discretecontinual finite elements, which are used for approximation of domain $\omega_{1} ;\left(N_{1}^{d c}-1\right)$ is the number of discrete-continual finite elements; $x_{1, i}^{f e}, i=1,2, \ldots, N_{1}^{f e}, x_{2, j}^{f e}, j=1,2, \ldots, N_{2}^{f e}$ are coordinates (along $x_{1}, x_{2}$ ) of nodes of finite elements, which are used for approximation of domain $\omega_{2} ;\left(N_{1}^{f e}-1\right)$ and $\left(N_{2}^{f e}-1\right)$ are numbers of finite elements along $x_{1}$ and $x_{2}$.

Two-index notation system is used for numbering of discrete-continual finite elements. Typical number of has the form $(k, i)$, where $k$ is the number of subdomain, $i$ is the number of element (along $x_{1}$ ). Three-index system is used for numbering of finite elements. Typical number of has the form $(k, i, j)$, where $k$ is the number of subdomain, $i$ and $j$ are numbers of elements (along $x_{1}$ and $x_{2}$ ). Let's $N_{1}^{f e}=N_{1}^{d c}=N_{1}$ and $x_{1, i}^{f e}=x_{1, i}^{d c}=x_{1, i}, i=1,2, \ldots, N_{1}$.

\section{Approximation models for subdomains and domain}

Discrete-continual approximation model within DCFEM presupposes mesh approximation for non-basic dimensions of extended domain (along $x_{1}$ ) while in the basic dimension (along $x_{2}$ ) problem remains continual. Thus extended subdomain $\omega_{1}$ is divided into discrete-continual finite elements

$$
\omega_{1}=\bigcup_{i=1}^{N_{1}-1} \omega_{1, i} ; \quad \omega_{1, i}=\left\{\left(x_{1}, x_{2}\right): x_{1, i}<x_{1}<x_{1, i+1}, \quad x_{2,1}^{b}<x_{2}<x_{2,2}^{b}\right\} .
$$

Flexural rigidity, Poisson's ratio and bedding value for discrete-continual finite element are defined by formulas:

$$
\bar{D}_{1, i}=\theta_{1, i} D_{1} ; \quad \bar{v}_{1, i}=\theta_{1, i} v_{1} ; \quad \bar{c}_{1, i}=\theta_{1, i} c_{1} ; \quad \theta_{1, i}=\left\{\begin{array}{ll}
1, & \omega_{1, i} \subset \Omega_{1} ; \\
0, & \omega_{1, i} \not \subset \Omega_{1} ;
\end{array} \quad D_{k}=\widetilde{E}_{k} h_{k}^{3} /\left[12\left(1-v_{k}^{2}\right)\right] ;\right.
$$

where $\theta_{1, i}$ is the characteristic function of element $\omega_{1, i} ; h_{k}$ is thickness of plate; $\widetilde{E}_{k}$ is the modulus of elasticity of material of plate. Let's $w_{1}$ be deflection of plate at subdomain $\omega_{1}$.

Basic nodal unknown functions are the following functions:

$$
y_{1}^{(1)}=y_{1}^{(1)}\left(x_{1}, x_{2}\right)=w_{1}\left(x_{1}, x_{2}\right) ; \quad y_{i}^{(1)}=y_{i}^{(1)}\left(x_{1}, x_{2}\right)=\partial_{2}^{i-1} w_{1}\left(x_{1}, x_{2}\right), \quad i=2,3,4 ;
$$




$$
z_{j}^{(1)}\left(x_{1}, x_{2}\right)=\partial_{1} y_{j}^{(1)}\left(x_{1}, x_{2}\right), j=1,2,3,4
$$

Thus, $y_{j}^{(1)}\left(x_{1}, x_{2}\right), j=1,2,3,4$ and $z_{j}^{(1)}\left(x_{1}, x_{2}\right), j=1,2,3,4$ are basic nodal unknown functions (superscript hereinafter corresponds to the number of considered subdomain i.e. $\left.\omega_{1}\right)$. Thus for node $(1, i)$ we have the following unknown functions: $y_{j}^{(1, i)}\left(x_{2}\right), j=1,2,3,4$ and $z_{j}^{(1, i)}\left(x_{2}\right), j=1,2,3,4$.

Polynomial (cubic) approximation along $x_{1}$ is used for $y_{j}^{(1, i)}\left(x_{2}\right), j=1,2,3,4$ within discrete-continual finite element. Approximation formulas for $z_{j}^{(1)}, j=1,2,3,4$ can be obtained after derivation in accordance with (4).

DCFEM is reduced at some stage to the solution of systems of $8 N_{1}$ first-order ordinary differential equations:

$$
\bar{Y}_{1}\left(x_{2}\right)=A_{1} \bar{Y}_{1}\left(x_{2}\right)+\overline{\widetilde{R}}_{1}\left(x_{2}\right)
$$

where $A_{1}$ is global matrix of coefficients of order $8 N_{1} ; \overline{\widetilde{R}}_{1}\left(x_{2}\right)$ is the right-side vector of order $8 N_{1} ; \bar{Y}_{1}\left(x_{2}\right)$ is global vector of nodal unknown functions (subscript corresponds to the number of subdomain $\omega_{1}$ ),

$$
\begin{gathered}
\bar{Y}_{1}=\bar{Y}_{1}\left(x_{2}\right)=\left[\begin{array}{lll}
\left(\bar{y}_{1,1}\right)^{T} & \left(\bar{y}_{1,2}\right)^{T}\left(\bar{y}_{1,3}\right)^{T} \quad\left(\bar{y}_{1,4}\right)^{T}
\end{array}\right]^{T} ; \\
\bar{y}_{1, j}=\bar{y}_{1, j}\left(x_{2}\right)=\left[\begin{array}{llll}
\left(\bar{y}_{n, j}^{(1,1)}\right)^{T} & \left(\bar{y}_{n, j}^{(1,2)}\right)^{T} & \ldots & \left(\bar{y}_{n, j}^{\left(1, N_{1}\right)}\right)^{T}
\end{array}\right]^{T}, j=1,2,3,4 ; \\
\bar{y}_{n, j}^{(1, i)}=\left[\begin{array}{lll}
y_{j}^{(1, i)} & z_{j}^{(1, i)}
\end{array}\right]^{T}, \quad j=1,2,3,4 ;
\end{gathered}
$$

Correct analytical solution of (6) is defined by formula

$$
\begin{gathered}
\bar{Y}_{1}\left(x_{2}\right)=E_{1}\left(x_{2}\right) \bar{C}_{1}+\bar{S}_{1}\left(x_{2}\right), \\
E_{1}\left(x_{2}\right)=\varepsilon_{1}\left(x_{2}-x_{2,1}^{b}\right)-\varepsilon_{1}\left(x_{2}-x_{2,2}^{b}\right), \quad \bar{S}_{1}\left(x_{2}\right)=\varepsilon_{1}\left(x_{2}\right) * \overline{\widetilde{R}}_{1}\left(x_{2}\right),
\end{gathered}
$$

where $\varepsilon_{1}\left(x_{2}\right)$ is the fundamental matrix-function of system (5), which is constructed in the special form convenient for problems of structural mechanics [1]; * is convolution notation; $\bar{C}_{1}$ is the vector of constants of order $8 N_{1}$.

Discrete approximation model within FEM for the considering two-dimensional problems presupposes finite element approximation along $x_{1}$ and $x_{2}$. Thus extended subdomain $\omega_{2}$ is divided into finite elements

$$
\omega_{2}=\bigcup_{i=1}^{N_{1}-1} \bigcup_{j=1}^{N_{2}-1} \omega_{2, i, j} ; \quad \omega_{2, i, j}=\left\{\left(x_{1}, x_{2}\right): x_{1, i}<x_{1}<x_{1, i+1}, \quad x_{2, j}^{f e}<x_{2}<x_{2, j+1}^{f e}\right\} .
$$

Flexural rigidity, Poisson's ratio and bedding value for finite element are defined by formulas: 


$$
\bar{D}_{2, i, j}=\theta_{2, i, j} D_{2} ; \quad \bar{v}_{2, i, j}=\theta_{2, i, j} v_{2} ; \quad \bar{c}_{2, i, j}=\theta_{2, i, j} c_{2} ; \quad \theta_{2, i, j}=\left\{\begin{array}{ll}
1, & \omega_{2, i, j} \subset \Omega_{2} \\
0, & \omega_{2, i, j} \not \subset \Omega_{2}
\end{array} ;\right.
$$

where $\theta_{2, i, j}$ is the characteristic function of element $\omega_{2, i, j}$,

Basic nodal unknowns are nodal values of function of deflection of plate and corresponding derivatives with respect to $x_{1}$ and $x_{2}$ (deflection angles), i.e. the following functions

$$
\begin{gathered}
w_{2}\left(x_{1}, x_{2}\right)=y_{1}^{(2)}\left(x_{1}, x_{2}\right) \\
\theta_{2,1}\left(x_{1}, x_{2}\right)=\partial_{2} w_{2}\left(x_{1}, x_{2}\right)=y_{2}^{(2)}\left(x_{1}, x_{2}\right) ; \theta_{2,2}\left(x_{1}, x_{2}\right)=-\partial_{1} w_{2}\left(x_{1}, x_{2}\right)=-z_{1}^{(2)}\left(x_{1}, x_{2}\right)
\end{gathered}
$$

Thus for node $(2, i, j)$ we have the following unknown functions: $y_{1}^{(2, i, j)}, y_{2}^{(2, i, j)}$ and $-z_{1}^{(2, i, j)}$.

Formula for approximation of deflection $w_{2}\left(x_{1}, x_{2}\right)$ within discrete-continual finite element $\omega_{2, i, j}$ has the form:

$$
\begin{aligned}
w_{2}\left(x_{1}, x_{2}\right) & =\alpha_{1}^{(2, i, j)}+\alpha_{2}^{(2, i, j)} x_{1}+\alpha_{3}^{(2, i, j)} x_{2}+\alpha_{4}^{(2, i, j)} x_{1}^{2}+\alpha_{5}^{(2, i, j)} x_{1} x_{2}+\alpha_{6}^{(2, i, j)} x_{2}^{2}+ \\
& +\alpha_{7}^{(2, i, j)} x_{1}^{3}+\alpha_{8}^{(2, i, j)} x_{1}^{2} x_{2}+\alpha_{9}^{(2, i, j)} x_{1} x_{2}^{2}+\alpha_{10}^{(2, i, j)} x_{2}^{3}+\alpha_{11}^{(2, i, j)} x_{1}^{3} x_{2}+\alpha_{12}^{(2, i, j)} x_{1} x_{2}^{3},
\end{aligned}
$$

where $\alpha_{p}^{(2, i, j)}, p=1,2, \ldots, 12$ are polynomial coefficients.

In other words, we find it convenient to use polynomials as form functions, which are defined by 12 coefficients (the fourth-order polynomials with several zero coefficients can be used). It should be noted that formula (17) has certain advantages. In particular, deflection $w_{2}\left(x_{1}, x_{2}\right)$ along line $x_{1}=$ const or line $x_{2}=$ const is described by cubic polynomial. All of the external boundaries and boundaries between the elements consists precisely of such lines. Since the third-order polynomial is uniquely defined by four coefficients, displacement along the boundary are uniquely determined by nodal displacements and nodal deflection angles at the ends of this boundary. Function $w_{2}\left(x_{1}, x_{2}\right)$ is continuous along any boundary between elements because values of polynomials at the ends of the boundary are the same for the adjacent elements. Besides, it can be noted that the gradient of function $w_{2}\left(x_{1}, x_{2}\right)$ with respect to normal to any boundary is described by third-order polynomial along this boundary (for instance, function $\partial_{1} w_{2}\left(x_{1}, x_{2}\right)$ along line $x_{1}=$ const $)$. Since we have only two given values of deflection angles at these lines, the third-order polynomial is ambiguously determined and deflection angle may be discontinuous (i.e. continuity of the first-order derivatives at boundaries between several finite elements is not provided). We have so-called nonconforming form function and nonconforming finite elements.

We should introduce additional nodal basic unknown, i.e. nodal value of function (mixed derivative)

$$
\tau^{(2)}\left(x_{1}, x_{2}\right)=\partial_{1} \partial_{2} w_{2}\left(x_{1}, x_{2}\right)=z_{2}^{(2)}\left(x_{1}, x_{2}\right)
$$

in order to obtain conforming finite elements. Corresponding formula instead of (17) has the following form

$$
w_{2}\left(x_{1}, x_{2}\right)=\bar{w}_{2}\left(x_{1}, x_{2}\right)+\alpha_{13}^{(2, i, j)} x_{1}^{3} x_{2}^{2}+\alpha_{14}^{(2, i, j)} x_{1}^{2} x_{2}^{3}+\alpha_{15}^{(2, i, j)} x_{1}^{2} x_{2}^{2}+\alpha_{16}^{(2, i, j)} x_{1}^{3} x_{2}^{3},
$$


where $\bar{w}_{2}\left(x_{1}, x_{2}\right)$ is defined by formula $(17) ; \alpha_{p}^{(2, i, j)}, p=1,2, \ldots, 16$ are polynomial coefficients.

As known, FEM is reduced to the solution of systems of $4 N_{1} N_{2}$ linear algebraic equations:

$$
K_{2} \bar{Y}_{2}=\bar{R}_{2}
$$

where $K_{2}$ is global stiffness matrix of order $4 N_{1} N_{2} ; \bar{R}_{2}$ is global right-side vector of order $4 N_{1} N_{2}$ (global load vector); $\bar{U}_{2}$ is global vector of nodal unknowns (subscript corresponds to the number of subdomain $\omega_{2}$ ),

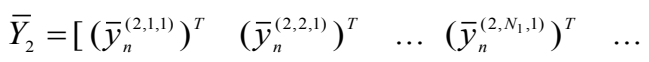

$$
\begin{aligned}
& \ldots\left(\bar{y}_{n}^{(2,1,2)}\right)^{T} \quad\left(\bar{y}_{n}^{(2,2,2)}\right)^{T} \quad \ldots\left(\bar{y}_{n}^{\left(2, N_{1}, 2\right)}\right)^{T} \quad \ldots \\
& \left.\ldots\left(\bar{y}_{n}^{\left(2,1, N_{2}\right)}\right)^{T} \quad\left(\bar{y}_{n}^{\left(2,2, N_{2}\right)}\right)^{T} \quad \ldots\left(\bar{y}_{n}^{\left(2, N_{1}, N_{2}\right)}\right)^{T}\right]^{T} \text {; } \\
& \bar{y}_{n}^{(2, i, j)}=\left[\begin{array}{llll}
y_{1}^{(2, i, j)} & z_{1}^{(2, i, j)} & y_{2}^{(2, i, j)} & z_{2}^{(2, i, j)}
\end{array}\right]^{T}, i=1,2, \ldots, N_{1}, j=1,2, \ldots, N_{2} .
\end{aligned}
$$

System (20) can be rewritten for all nodes with indexes $1<j<N_{2}$ (i.e. $\left.x_{2,2}^{b}<x_{2}<x_{2,3}^{b}\right)$ in the following form (resolving system of $4 N_{1}\left(N_{2}-2\right)$ linear algebraic equations):

$$
\widetilde{K}_{2} \bar{Y}_{2}=\overline{\widetilde{R}}_{2}
$$

where $\widetilde{K}_{2}$ is reduced global stiffness matrix of size $\left[4 N_{1}\left(N_{2}-2\right)\right] \times\left[4 N_{1} N_{2}\right] ; \overline{\widetilde{R}}_{2}$ is reduced right-side vector of order $4 N_{1}\left(N_{2}-2\right)$.

The Reported study was Funded by Government Program of the Russian Federation "Development of science and technology" (2013-2020) within Program of Fundamental Researches of Ministry of Construction, Housing and Utilities of the Russian Federation and Russian Academy of Architecture and Construction Sciences, the Research Projects 7.1.1, 7.1.2, 7.4.2, 7.4.3, 7.4.17”.

\section{References}

1. P.A. Akimov, Applied Mechanics and Materials 204-208, 4502-4505 (2012)

2. P.A. Akimov, M.L. Mozgaleva, Applied Mechanics and Materials 580-583, 2898-2902 (2014)

3. P.A. Akimov, O.A. Negrozov, Procedia Engineering 153, 8-15 (2016)

4. P.A. Akimov, O.A. Negrozov, Procedia Engineering 153, 16-23 (2016)

5. P.A. Akimov, O.A. Negrozov, Procedia Engineering 153, 24-31 (2016)

6. E.M. Alawadhi, Finite Element Simulations Using ANSYS (CRC Press, 2009)

7. H. Assaee, N. Pournoori, Thin-Walled Structures 124, 430-436 (2018)

8. E.J. Barbero, Finite Element Analysis of Composite Materials Using ANSYS (CRC Press, 2013)

9. K.-J. Bathe, Finite Element Procedures (Prentice Hall Inc., 1996) 
10. V.G. Belardi, P. Fanelli, F. Vivio, V. G. Belardi, Composites Part B: Engineering 140, 250-259 (2018)

11. A. Belostotsky, T. Kaytukov, I. Afanasyeva, J. Grosel, MATEC Web Conf. 2017 116, 00016 (2017)

12. U. Bohinc, B. Brank, A. Ibrahimbegovics, Computer Methods in Applied Mechanics and Engineering 269, 415-436 (2014)

13. E. Burman, P. Hansbo, M.G. Larson, Finite Elements in Analysis and Design 142, 5160 (2018)

14. E. Carrera, M. Cinefra, G. Li, Composite Structures 183, 63-76 (2018)

15. J. Chen, Journal of Computational and Applied Mathematics 329, 68-83 (2018)

16. E.H. Dill, The Finite Element Method for Mechanics of Solids with ANSYS Applitions (CRC Press, 2011)

17. P. Gehlot, A.K. Sharma, A.S. Rajawat, Materials Today: Proceedings 5(2), Part 1, 5145-5153 (2018)

18. M. Ghorashi, International Journal of Mechanical Sciences 136, 313-320 (2018)

19. S. Goswami, W. Becker, World Journal of Mechanics 3, 194-201 (2013)

20. R.J. Hanson, T. Hopkins, Numerical Computing with Modern Fortran (Applied Mathematics) (SIAM-Society for Industrial and Applied Mathematics, 2013)

21. P.-F. Hou, J.-Y. Chen, Engineering Analysis with Boundary Elements 93, 10-20 (2018)

22. A.V. Ignatyev, V.A. Ignatyev, Procedia Engineering 150, 1766-1770 (2016)

23. M. Karkon, M. Rezaiee-Pajand, Applied Mathematical Modelling 41, 618-629 (2017)

24. A.T. Karttunen, R. von Hertzen, J.N. Reddy, J. Romanoff, Computers \& Structures 200, 21-31 (2018)

25. M. Khezri, M. Abbasi, K.J.R. Rasmussen, Thin-Walled Structures 111, 113-125 (2017)

26. J. Li, Z. Shi, L. Liu, Computers \& Structures 202, 105-128 (2018)

27. R. Li, Y. Tian, X. Zheng, H. Wang, S. Xiong, B. Wang, European Journal of Mechanics - A/Solids 66, 103-113 (2017)

28. R. Li, P. Wang, R. Xue, X. Guo, International Journal of Mechanical Sciences 131132, 179-190 (2017)

29. L. Lyakhovich, O. Negrozov, MATEC Web of Conferences 117, 00109 (2017)

30. L. Lyakhovich, O. Negrozov, MATEC Web of Conferences 117, 00110 (2017)

31. E. Madenci, I. Guven, The Finite Element Method and Applications in Engineering Using ANSYS (Springer, 2015)

32. F. Millar, D. Mora, Journal of Computational and Applied Mathematics 286, 68-78 (2015)

33. S. Moaveni, Finite Element Analysis: Theory and Application with ANSYS (Prentice Hall, 2014)

34. L. Najarzadeh, B. Movahedian, M. Azhari, Thin-Walled Structures 123, 36-47 (2018).

35. O.A. Negrozov, P.A. Akimov, I.Yu. Lantsova, Procedia Engineering 153, 926-932 (2016)

36. H. Nguyen-Xuan, Computers \& Structures 188, 45-62 (2017)

37. A. Pandey, A. Arockiarajan, International Journal of Mechanical Sciences 130, 416425 (2017)

38. M. Park, D.-H. Choi, Applied Mathematical Modelling 61, 49-71 (2018) 
39. A.S. Rajawat, A.K. Sharma, P. Gehlot, Materials Today: Proceedings 5(2), Part 1, 5313-5321 (2018)

40. I. Senjanovic, N. Vladimir, D.S. Cho, International Journal of Naval Architecture and Ocean Engineering 7(2), 324-345 (2015)

41. A.I. Shirkol, T. Nasar, Journal of Ocean Engineering and Science 3(1), 19-37 (2018)

42. F. Wang, M. Avci, Y. An, Journal of Mathematical Analysis and Applications 409(1), $140-146$ (2014)

43. F. Wu, W. Zeng, L.Y. Yao, G.R. Liu, Applied Mathematical Modelling 53, 333-352 (2018)

44. O.C. Zienkiewicz, R.L. Taylor, D.D. Fox, The Finite Element Method for Solid and Structural Mechanics (Butterworth-Heinemann, 2013)

45. O.C. Zienkiewicz, R.L. Taylor, J.Z. Zhu, The Finite Element Method: Its Basis and Fundamentals (Butterworth-Heinemann, 2005)

46. A.B. Zolotov, P.A. Akimov, Proceedings of the International Symposium LSCE 2002 organized by Polish Chapter of IASS (Warsaw, Poland, 2002) 